\title{
Repetitive Painting (REPEAT) Irradiation in Stereotactic Radiotherapy Using Helical Tomotherapy
}

\author{
YU OHKUBO ${ }^{1}$, TAKUYA KUMAZAWA ${ }^{1}$, RYUTA HIRAI ${ }^{2}$ and SHIN-EI NODA ${ }^{2}$ \\ ${ }^{1}$ Department of Radiation Oncology, Saku Central Hospital Advanced Care Center, Nagano, Japan; \\ ${ }^{2}$ Department of Radiation Oncology, Saitama Medical University International Medical Center, Saitama, Japan
}

\begin{abstract}
Background/Aim: Stereotactic radiotherapy (SRT) for spine metastases with helical tomotherapy requires a long irradiation time due to the high dose per fraction. Since helical tomotherapy can neither confirm nor correct the position during irradiation, a plan with a long irradiation time cannot be used in actual clinical practice, given the intra-fractional motion error. To address this problem, we devised a method called REPEAT irradiation. Patients and Methods: REPEtitive pAinTing (REPEAT) irradiation is a method of dividing the irradiation for a given fraction per day into several sessions and performing the irradiation after position correction using mega-voltage computed tomography images for each session. In order to evaluate how REPEAT irradiation changes irradiation time and the dose-volume histogram (DVH), a planning study with helical tomotherapy was conducted using CT images of a patient with lumbar spine metastasis. Results: In this case, we found that dividing 3 irradiation fractions into 3 sessions per day (i.e., 9 fractions $=9$ sessions in 3 days) using REPEAT irradiation shortened the irradiation time per session and simultaneously improved dose-volume histogram parameters. Conclusion: Although the optimal number of sessions may differ depending on the patient's condition, the fixing method, the irradiation site, and the calculation parameters, REPEAT irradiation does not require any special equipment and is a simple practical treatment method.
\end{abstract}

Stereotactic radiotherapy (SRT) is a method of concentrating doses of radiation on small lesions using the modern

This article is freely accessible online.

Correspondence to: $\mathrm{Yu}$ Ohkubo, Department of Radiation Oncology, Saku Central Hospital Advanced Care Center, 3400-28 Nakagomi, Saku-shi, Nagano 385-0051, Japan. Tel: +81 267628181, Fax: +81 267887354, e-mail: ohkuboy@gmail.com

Key Words: Stereotactic radiotherapy, helical tomotherapy, intrafractional motion, image-guided radiotherapy (IGRT). radiation technique. This technology has become widespread as it can be used not only for special treatment devices such as Gamma Knife and CyberKnife, but also for general purpose linear accelerators. SRT is one of the standard treatments for localized tumors such as metastatic brain tumors and early-stage lung cancer (1-3). Recently, the effectiveness of SRT for oligometastases has also been reported (4). In addition, research on the combination therapy of SRT and immunotherapy is progressing and the need for SRT is anticipated to further increase (5).

Bone metastases are frequently distant metastases, typically treated with palliative irradiation of 8-30 Gy. However, with the development of chemotherapy and immunotherapy (e.g., PD-1/PD-L1 inhibitors), long-term survival has become a possibility (6-8). Some studies have found that increasing the prescribed dose with SRT may improve quality of life (QOL) through long-term control of vertebral metastases $(9,10)$, while others have demonstrated the validity of SRT techniques for vertebral metastases, including re-irradiation cases $(11,12)$. Still others have investigated the differences in vertebral body SRT depending on treatment devices such as CyberKnife, volumetric-modulated arc therapy (VMAT), and helical tomotherapy $(13,14)$.

Helical tomotherapy is an intensity modulated radiotherapy (IMRT)-dedicated device and is characterized by helical irradiation using a binary multi-leaf collimator (MLC) that can obtain a highly uniform dose distribution. Helical irradiation, on the other hand, is disadvantageous in that the irradiation time is long when the irradiation range is wide in the longitudinal direction. Irradiation time is particularly lengthy in SRT, for which a higher dose of Xray is required per fraction for irradiation. Another drawback is that the position of the target cannot be confirmed and corrected during irradiation. Past planning studies for spinal metastases using helical tomotherapy have reported that an irradiation time of 20-30 min is required $(13,15)$. Previously, we reported that spine position should be checked every 4-6 min in order to ensure irradiation accuracy within the millimeter or submillimeter range in spine SRT (16). This is achievable with treatment devices such as CyberKnife, 


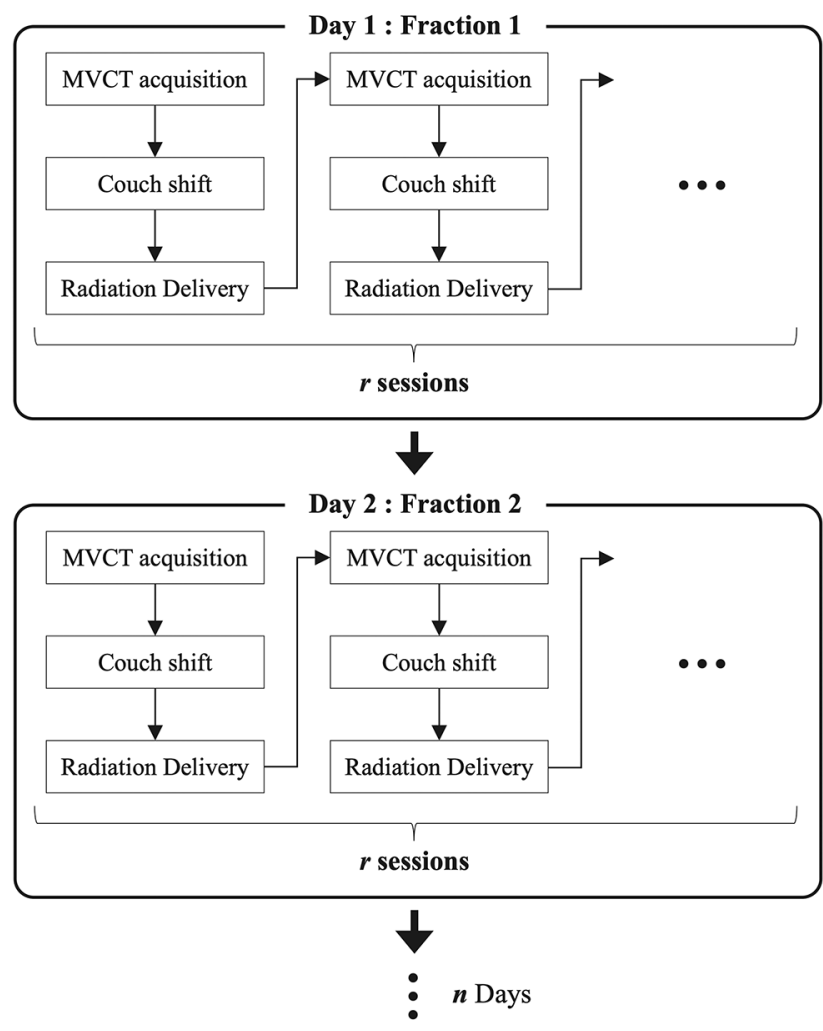

Fractionation Schedule: $(\boldsymbol{r} \times \boldsymbol{n})$ sessions in $\boldsymbol{n}$ fractions ( $\boldsymbol{n}$ days)

Figure 1. Concept diagram of REPEAT irradiation. REPEAT irradiation is a method which divides the irradiation administered in each fraction into several sessions. The $r$ number can be set according to the allowable irradiation time. Position correction with MVCT images is performed at each session. With daily treatment (=r sessions), the prescribed dose for each fraction per day is delivered. REPEAT, REPEtitive pAinTing; MVCT, Megavoltage computed tomography.

which can correct the position by tracking during irradiation. However, as tomotherapy cannot correct the position during irradiation, the planning target volume (PTV) margin must be widened in consideration of intra-fractional motion error. In cases for which the clinical target volume (CTV) and spinal cord are close to each other in spinal SRT, this may result in inadequate treatment with poor target coverage.

In this study, we propose a method called Repetitive painting (REPEAT) irradiation to solve this problem.

\section{Patients and Methods}

The concept of REPEAT irradiation. REPEtitive pAinTing (REPEAT) irradiation is a method of dividing the daily fraction of irradiation into several sessions and performing the irradiation after position correction is performed before each session using mega-voltage computed tomography (MVCT) images. The concept diagram for this is shown in Figure 1. For example, if $24 \mathrm{~Gy}$ of irradiation is administered in 3 fractions (i.e., in 3 days), assume that $10 \mathrm{~min}$ are needed to deliver 8
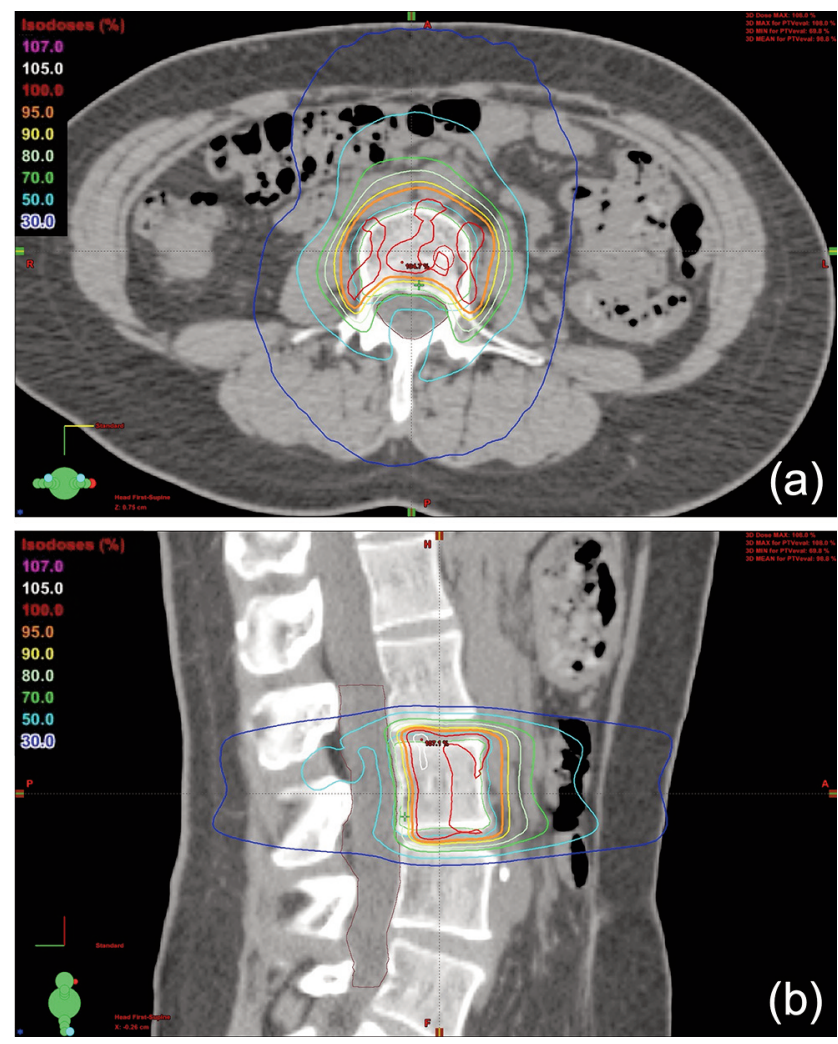

Figure 2. Dose distribution of stereotactic irradiation for lumbar spine metastasis in our planning study. Axial (a) and sagittal (b) sections of $C T$ slices. The thin red line represents gross tumor volume (GTV); the thin green line represents clinical target volume (CTV); the thin cyan line represents planning target volume (PTV); and the thin brown line represents the thecal sac.

Gy in a day. When performing 2 sessions per day using REPEAT irradiation, 8 Gy irradiation is divided into 2 parts: 4 Gy irradiation after MVCT-based patient set-up, followed by another position correction by MVCT, and then one more irradiation of $4 \mathrm{~Gy}$, resulting in 8 Gy irradiation per fraction (per day), spread out over 2 sessions. Simply put, the 4 Gy irradiation in one session is half of $8 \mathrm{~Gy}$, and can be delivered in $5 \mathrm{~min}$. While it is possible to increase the number of sessions to two, three, four, etc., depending on the allowable irradiation time per session, when the necessary time for position correction using MVCT is added to each session, the total treatment time can get lengthy.

Planning study. In order to evaluate how REPEAT irradiation changes irradiation time and the dose-volume histogram (DVH), a planning study was conducted using CT images of a patient with lumbar spine metastasis (Figure 2). This case was for demonstration purposes only, and the patient was not actually treated with helical tomotherapy.

Eclipse treatment planning system (version 11.0, Varian Medical Systems, Palo Alto, CA, USA) was used for contouring, and TomoTherapy Planning Station (TomoHDA Version 2.1.6, Accuray, Sunnyvale, CA, USA) was used for dose calculation. With the assistance of magnetic resonance imaging (MRI) and computed tomography (CT), 

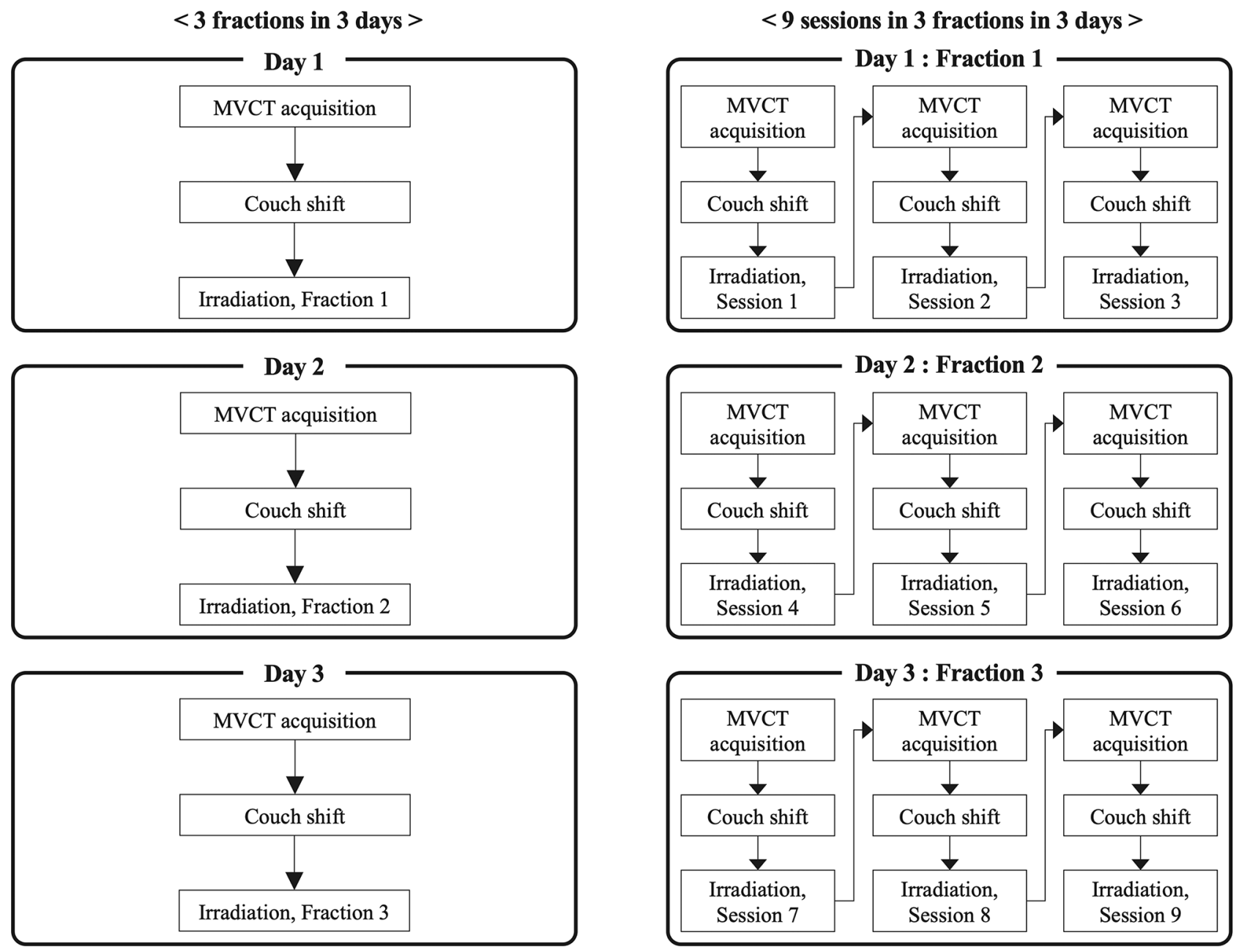

Figure 3. Treatment flow in a typical irradiation regimen (left column) as compared to REPEAT irradiation administered over 9 sessions (right column). REPEAT, REPEtitive pAinTing; MVCT, megavoltage computed tomography.

the CTV contouring was done according to the ASTRO guidelines and agreed upon by two radiation oncologists (17). A 2-mm margin was added to the CTV to create the PTV, with the PTV excluding the thecal sac defined as PTV $_{\text {evl }}$. The prescribed dose (PD) was $24 \mathrm{~Gy}$, administered in 3 fractions (over 3 days) for $50 \%$ of the $\mathrm{PTV}_{\text {evl }}$ volume $\left(\mathrm{D}_{50 \%}\right)$. Dose constraints were as follows: dose to $95 \%$ volume to the PTV $_{\text {evl }}\left(D_{95 \%}\right) \geq 70-80 \%$ of PD; maximum dose to PTV evl $\left(D_{\max }\right) \leq 140 \%$ of PD; $\mathrm{D}_{\max }$ of thecal sac $<20.3 \mathrm{~Gy}$; dose irradiated to the $0.35 \mathrm{~cm}^{3}$ of thecal sac $\left(\mathrm{D}_{0.35 \mathrm{cc}}\right)<18 \mathrm{~Gy}$; dose irradiated to $1 \mathrm{~cm}^{3}$ of intestine $\left(\mathrm{D}_{1 \mathrm{cc}}\right)<20 \mathrm{~Gy} ; \mathrm{D}_{1 \mathrm{cc}}$ of the skin<26 Gy; mean dose to unilateral kidney $\left(\mathrm{D}_{\text {mean }}\right)<5 \mathrm{~Gy}$; and $\mathrm{D}_{\text {mean }}$ of bilateral kidney $<9$ Gy $(18,19)$.

In order to avoid intentional inverse-planning, calculation parameters were fixed and only 100 iterations were performed in the TomoTherapy Planning Station. Field width (FW), Jaw Mode, and Pitch were fixed, and only the Modulation Factor (MF) and the number of fractions were changed for comparison. Since normalization is not possible with the TomoTherapy Planning Station, the final DVH parameters were normalized to be PTVevl $\mathrm{D}_{50 \%}=24.0 \mathrm{~Gy}$ and evaluated in Eclipse. In total, 9 patterns were calculated by changing the conditions of MF to $1.5,2.0$, and 2.5 and the number of fractions to 3 fractions without REPEAT irradiation ( 3 fractions over 3 days), 6 fractions with 2 sessions per day using REPEAT irradiation (i.e., 6 sessions over 3 days), and 9 fractions with 3 sessions per day using REPEAT irradiation (i.e., 9 sessions over 3 days). Figure 3 shows the treatment flow without REPEAT irradiation and with REPEAT irradiation for 9 sessions in 3 days.

The appropriate irradiation time per session differs based on a patient's general condition and immobilization method. In the present study, we considered $6 \mathrm{~min}$ or less to be an acceptable irradiation time. In our evaluation, we compared $\mathrm{D}_{95 \%}$ of $\mathrm{PTV}_{\mathrm{evl}}$, $\mathrm{D}_{\max }$ of thecal sac, and $\mathrm{D}_{1 \mathrm{cc}}$ of the intestinal tract of each plan.

\section{Results}

Our results are presented in Table I. Increasing the MF (the degree of intensity modulation) resulted in a correlative increase in irradiation time. Irradiation administered in 3 
Table I. Dose volume histogram analysis.

\begin{tabular}{|c|c|c|c|c|c|c|c|c|c|c|}
\hline \multicolumn{5}{|c|}{ Calculation conditions } & \multirow[b]{2}{*}{ IT (min) } & \multirow[b]{2}{*}{$\mathrm{IT} \leq 6 \mathrm{~min}$} & \multicolumn{2}{|c|}{$\mathrm{PTV}_{\mathrm{evl}}$} & \multirow{2}{*}{$\begin{array}{l}\text { Thecal sac } \\
\mathrm{D}_{\max }(\mathrm{Gy})\end{array}$} & \multirow{2}{*}{$\frac{\text { Intestine }}{\mathrm{D}_{1 \mathrm{cc}}(\mathrm{Gy})}$} \\
\hline $\mathrm{FW}(\mathrm{cm})$ & Jaw mode & Pitch & Fractions & $\mathrm{MF}$ & & & $\mathrm{D}_{50 \%}(\mathrm{~Gy})$ & $\mathrm{D}_{95 \%}(\mathrm{~Gy})$ & & \\
\hline \multirow[t]{9}{*}{1.048} & Fixed & 0.287 & $3^{* 1}$ & 1.5 & 10.8 & No & 24.00 & 20.63 & 18.48 & 18.21 \\
\hline & & & & 2.0 & 14.5 & No & 24.00 & 21.03 & 17.78 & 18.14 \\
\hline & & & & 2.5 & \multicolumn{6}{|c|}{ The calculated gantry period exceeds the maximum available gantry period ( 60 seconds) } \\
\hline & & & $6^{* 2}$ & 1.5 & 5.5 & Yes & 24.00 & 20.62 & 18.45 & 18.21 \\
\hline & & & & 2.0 & 7.3 & No & 24.00 & 21.02 & 17.85 & 18.13 \\
\hline & & & & 2.5 & 9.1 & No & 24.00 & 21.09 & 17.75 & 18.05 \\
\hline & & & $9 * 3$ & 1.5 & 3.7 & Yes & 24.00 & 20.61 & 18.39 & 18.20 \\
\hline & & & & 2.0 & 4.9 & Yes & 24.00 & 21.01 & 17.82 & 18.12 \\
\hline & & & & 2.5 & 6.1 & No & 24.00 & 21.07 & 17.73 & 18.04 \\
\hline
\end{tabular}

FW, Field width; MF, modulation factor; IT, irradiation time per fraction; $\mathrm{PTV}_{\text {evl }}$, evaluated planning target volume; $\mathrm{D}_{50 \%}$, the dose to 50\% volume; $\mathrm{D}_{95 \%}$, the dose to $95 \%$ volume; $\mathrm{D}_{\max }$, maximum point dose; $\mathrm{D}_{1 \mathrm{cc}}$, the dose to the hottest $1 \mathrm{~cm}^{3} .{ }^{* 1}$ One session per day, ${ }^{* 2}$ Two sessions per day, *3 Three sessions per day.

fractions without REPEAT irradiation yielded irradiation times of 10.8 and $14.5 \mathrm{~min}$ for MF 1.5 and 2.0, respectively. When MF was 2.5, irradiation was not possible due to the limitation of the gantry rotation speed; namely, the calculated gantry period exceeded the maximum available gantry period. Therefore, without REPEAT irradiation, irradiation time either exceeded 6 min or irradiation was not an option, regardless of the MF setting. With REPEAT irradiation, the irradiation time could be kept within 6 min by dividing the irradiation into 2 or 3 sessions with an MF of 1.5 , or by dividing the irradiation into 3 sessions with an MF of 2.0.

When REPEAT irradiation was used, we observed nearly no difference in DVH parameters between 2 sessions per day ( 6 fractions $=6$ sessions in 3 days) and 3 sessions per day $(9$ fractions $=9$ sessions in 3 days) for MF 1.5. However, when MF was increased to 2.0, we observed improved tumor coverage $\left(\mathrm{PTV}_{\text {evl }} \mathrm{D}_{95 \%}\right)$ and lower risk organ dose $\left(\mathrm{D}_{\max }\right.$ of thecal sac and $\mathrm{D}_{1 \mathrm{cc}}$ of intestine). Based on the assumption that an irradiation time $\leq 6 \mathrm{~min}$ is acceptable, treatment with MF 2.0 in 3 sessions per day was considered optimal under these conditions.

\section{Discussion}

A longer irradiation time gives rise to a larger intra-fractional motion error, which requires a larger PTV margin (16). When REPEAT irradiation is used, the irradiation time per session is shortened, allowing for reduction in the PTV margin (or irradiation can be performed with small PTV margins). This would theoretically allow for better dose coverage of the target while also lowering the dose to the organs at risk. In addition, by increasing the number of sessions (reducing the dose delivered per session), irradiation time for each session is shortened, and thus a better plan may be created by increasing the degree of intensity modulation. This planning study revealed that it is possible to reduce the dose to risk organs and improve dose coverage of the target $\left(\mathrm{PTV}_{\mathrm{evl}} \mathrm{D}_{95 \%}\right)$ by increasing the intensity modulation with higher MF. In terms of field width, Aljabab et al. used a field width of $2.5 \mathrm{~cm}$ because the irradiation time is 2-3 times longer with a field width of $1 \mathrm{~cm}$ in spine SRT (20). However, when the tilt of the vertebral body is large, a wider field width may worsen the dose distribution. REPEAT irradiation could provide better dose distribution because it can use a small field width, especially for SRT of the cervical or upper thoracic spine.

In previous studies comparing various irradiation modalities such as CyberKnife and VMAT, superiority of tomotherapy was reported, especially for targets with complex shapes $(13,14)$. On the other hand, unlike CyberKnife (which allows tracking during irradiation), tomotherapy basically does not allow for position confirmation or correction during irradiation. Therefore, irradiation time is important, especially for SRT with high dose irradiation. For spine SRT using tomotherapy, several studies have reported the following irradiation times: irradiating 16 Gy in 1 fraction in an average of $29 \mathrm{~min}$ and 19 seconds, 33 Gy in 3 fractions (11 Gy per fraction) in 29.17 min, and 40 Gy in 5 fractions (8 Gy per fraction) in 20.17 min $(13,15)$. However, considering intra-fractional motion error, it is difficult to use these in actual clinical practice.

In tomotherapy, the rotation speed of the gantry cannot be reduced to less than $60 \mathrm{sec} /$ rotation due to the limitations of the machine. Accordingly, Aljabab et al. reported that in spine SRT, when the gantry period exceeded $60 \mathrm{sec} /$ rotation, irradiation was divided into two parts (20). However, even with that setup, irradiation time still exceeded $10 \mathrm{~min}$. Therefore, the number of sessions must be increased more 
aggressively and the irradiation time per session must be shortened. The concept of REPEAT irradiation can be applied not only to tomotherapy but also to VMAT, specifically by increasing the number of arcs and performing image-guided radiotherapy between them. This study investigated SRT of the vertebral body, but the concept of REPEAT irradiation can be applied to other regions by adjusting the number of sessions according to the intrafractional motion error for each region.

The disadvantages of REPEAT irradiation include the increase in exposure dose with the higher number of MVCT images, and the increase in total treatment time. Although the exposure dose increases with the frequency of MVCTbased positioning, the total number of MVCT images remains low because REPEAT irradiation is a method used in SRT that requires only a small number of irradiations with a high dose per fraction. For example, compared to the 20 times of MVCT required for administering $60 \mathrm{~Gy}$ in 20 fractions for prostate cancer, the required frequency of MVCT-based positioning is much less for REPEAT irradiation. Given the benefits of highly accurate treatment, this is considered to be an acceptable increase in radiation exposure. Another disadvantage is that the number of MVCT-based positioning increases with the number of sessions, resulting in longer total treatment time. In patients with bone metastases and severe pain, a long total treatment time may cause more pain and make treatment impossible. In this case, it is possible to perform irradiation with a break between sessions. Alternatively, the number of REPEAT irradiation sessions could be reduced by expanding the PTV margin and increasing the permissible irradiation time.

One limitation of the study is that the optimal irradiation time per session is unknown. In a previous study targeting patients who received SBRT using CyberKnife, we reported that spine position should be checked every 4-6 min to ensure irradiation accuracy within the millimeter or submillimeter range in spine SRT (16). However, the amount of deviation of the target during irradiation may differ depending on the degree of pain experienced by the patient, the fixing method, and the irradiation site. Moreover, the permissible irradiation time for each session changes depending on the PTV margin. Accordingly, each facility will need to consider and set an appropriate irradiation time based on their treatment protocol. In addition, in the second and subsequent sessions, the amount of deviation may not be the same as in the first session, and there is a possibility that the amount of deviation may become larger as time passes from the start of irradiation. In this regard, REPEAT irradiation provides the amount of position correction from MVCT imaging between sessions. The accumulation of such data will provide useful information for determining whether the current PTV margin and irradiation time per session at a facility is appropriate, and aid in setting more appropriate irradiation conditions.
REPEAT irradiation requires no special equipment and simply increases the number of fractions (i.e., sessions) to shorten the irradiation time per session, while utilizing MVCT images to confirm and correct the position for each session. This allows the PTV margin to remain small, reduces the risk of adverse events, and may increase the probability of tumor control. REPEAT irradiation is a remarkably simple but practical treatment method.

\section{Conflicts of Interest}

The Authors declare that there are no conflicts of interest.

\section{Authors' Contributions}

Conception and study methodology: YO, RH, and SN. Data collection and analysis: YO. Interpretation of the data: YO, RH, TK, and SN. Drafting the work: YO. Review and supervision: RH, TK, and SN. Final approval of the version to be published: All Authors.

\section{Acknowledgements}

This study was approved by the Institutional Review Board of Saku Central Hospital Advanced Care Center (Authorization number: R201902-05).

\section{References}

1 Tandberg DJ, Tong BC, Ackerson BG and Kelsey CR: Surgery versus stereotactic body radiation therapy for stage I non-small cell lung cancer: A comprehensive review. Cancer 124(4): 667678, 2018. PMID: 29266226. DOI: 10.1002/cncr.31196

2 Kwon AK, Dibiase SJ, Wang B, Hughes SL, Milcarek B and Zhu Y: Hypofractionated stereotactic radiotherapy for the treatment of brain metastases. Cancer 115(4): 890-898, 2009. PMID: 19132728. DOI: 10.1002/cncr.24082

3 Teshima T, Numasaki H, Nishio M, Ikeda H, Sekiguchi K, Kamikonya N, Koizumi M, Tago M, Ando Y, Tsukamoto N, Terahara A, Nakamura K, Murakami M, Takahashi M, Nishimura T and Japanese Society for Therapeutic Radiology and Oncology Database Committee: Japanese structure survey of radiation oncology in 2009 based on institutional stratification of the Patterns of Care Study. J Radiat Res 53(5): 710-721, 2012. PMID: 22843366. DOI: $10.1093 / \mathrm{jrr} / \mathrm{rrs} 028$

4 Palma DA, Olson R, Harrow S, Gaede S, Louie AV, Haasbeek C, Mulroy L, Lock M, Rodrigues GB, Yaremko BP, Schellenberg D, Ahmad B, Griffioen G, Senthi S, Swaminath A, Kopek N, Liu M, Moore K, Currie S, Bauman GS, Warner A and Senan S: Stereotactic ablative radiotherapy versus standard of care palliative treatment in patients with oligometastatic cancers (SABR-COMET): a randomised, phase 2, open-label trial. Lancet 393(10185): 2051-2058, 2019. PMID: 30982687. DOI: 10.1016/S0140-6736(18)32487-5

5 Kroeze SG, Fritz C, Hoyer M, Lo SS, Ricardi U, Sahgal A, Stahel R, Stupp R and Guckenberger M: Toxicity of concurrent stereotactic radiotherapy and targeted therapy or immunotherapy: A systematic review. Cancer Treat Rev 53: 2537, 2017. PMID: 28056412. DOI: 10.1016/j.ctrv.2016.11.013 
6 Cardoso F, Harbeck N, Fallowfield L, Kyriakides S, Senkus E and ESMO Guidelines Working Group: Locally recurrent or metastatic breast cancer: ESMO Clinical Practice Guidelines for diagnosis, treatment and follow-up. Ann Oncol 23 Suppl 7: vii11-vii19, 2012. PMID: 22997442. DOI: 10.1093/annonc/ mds 232

7 Ettinger DS, Wood DE, Aisner DL, Akerley W, Bauman J, Chirieac LR, D'Amico TA, DeCamp MM, Dilling TJ, Dobelbower M, Doebele RC, Govindan R, Gubens MA, Hennon M, Horn L, Komaki R, Lackner RP, Lanuti M, Leal TA, Leisch LJ, Lilenbaum R, Lin J, Loo BW Jr, Martins R, Otterson GA, Reckamp K, Riely GJ, Schild SE, Shapiro TA, Stevenson J, Swanson SJ, Tauer K, Yang SC, Gregory K and Hughes M: Nonsmall cell lung cancer, version 5.2017, NCCN clinical practice guidelines in oncology. J Natl Compr Canc Netw 15(4): 504535, 2017. PMID: 28404761. DOI: 10.6004/jncen.2017.0050

8 Gubens MA and Davies M: NCCN guidelines updates: New immunotherapy strategies for improving outcomes in non-small cell lung cancer. J Natl Compr Canc Netw 17(5.5): 574-578, 2019. PMID: 31117034. DOI: 10.6004/jncen.2019.5005

9 Kumar R, Nater A, Hashmi A, Myrehaug S, Lee Y, Ma L, Redmond K, Lo SS, Chang EL, Yee A, Fisher CG, Fehlings MG and Sahgal A: The era of stereotactic body radiotherapy for spinal metastases and the multidisciplinary management of complex cases. Neurooncol Pract 3(1): 48-58, 2016. PMID: 31579521. DOI: 10.1093/nop/npv022

10 Zeng KL, Tseng CL, Soliman H, Weiss Y, Sahgal A and Myrehaug S: Stereotactic body radiotherapy (SBRT) for oligometastatic spine metastases: an overview. Front Oncol 9: 337, 2019. PMID: 31119099. DOI: 10.3389/fonc.2019.00337

11 Kawashiro S, Harada H, Katagiri H, Asakura H, Ogawa H, Onoe T, Sumita K, Murayama S, Murata H, Nemoto K, Takahashi M and Nishimura T: Reirradiation of spinal metastases with intensity-modulated radiation therapy: an analysis of 23 patients. J Radiat Res 57(2): 150-156, 2016. PMID: 26662113. DOI: $10.1093 / \mathrm{jrr} / \mathrm{rrv} 083$

12 Myrehaug S, Sahgal A, Hayashi M, Levivier M, Ma L, Martinez R, Paddick I, Régis J, Ryu S, Slotman B and De Salles A: Reirradiation spine stereotactic body radiation therapy for spinal metastases: systematic review. J Neurosurg Spine 27(4): 428435, 2017. PMID: 28708043. DOI: 10.3171/2017.2.SPINE16976

13 Yang J, Ma L, Wang XS, Xu WX, Cong XH, Xu SP, Ju ZJ, Du $\mathrm{L}$, Cai BN and Yang J: Dosimetric evaluation of 4 different treatment modalities for curative-intent stereotactic body radiation therapy for isolated thoracic spinal metastases. Med Dosim 41(2): 105-112, 2016. PMID: 26831753. DOI: 10.1016/ j.meddos.2015.10.003
14 Kim J, Jang HS, Kim YS, Choi BO and Kang YN: Comparison of spinal Stereotactic Body Radiotherapy (SBRT) planning techniques: intensity-modulated radiation therapy, modulated arc therapy, and helical tomotherapy. Med Dosim 42(3): 210-215, 2017. PMID: 28526192. DOI: 10.1016/j.meddos.2017.04.001

15 Gallo JJ, Kaufman I, Powell R, Pandya S, Somnay A, Bossenberger T, Ramirez E, Reynolds R, Solberg $\mathrm{T}$ and Burmeister J: Single-fraction spine SBRT end-to-end testing on TomoTherapy, Vero, TrueBeam, and CyberKnife treatment platforms using a novel anthropomorphic phantom. J Appl Clin Med Phys 16(1): 5120, 2015. PMID: 25679169. DOI: 10.1120/ jacmp.v16i1.5120

16 Hirai R, Ohkubo YU, Igari M, Kumazaki YU, Aoshika T, Ryuno Y, Saito S, Abe T, Noda SE and Kato S: Time dependence of intra-fractional motion in spinal stereotactic body radiotherapy. In Vivo 35(4): 2433-2437, 2021. PMID: 34182527. DOI: 10.21873/invivo.12521

17 Cox BW, Spratt DE, Lovelock M, Bilsky MH, Lis E, Ryu S, Sheehan J, Gerszten PC, Chang E, Gibbs I, Soltys S, Sahgal A, Deasy J, Flickinger J, Quader M, Mindea S and Yamada Y: International Spine Radiosurgery Consortium consensus guidelines for target volume definition in spinal stereotactic radiosurgery. Int J Radiat Oncol Biol Phys 83(5): e597-e605, 2012. PMID: 22608954. DOI: 10.1016/j.ijrobp.2012.03.009

18 Ito K, Furuya T, Shikama N, Nihei K, Tanaka H, Kumazaki Y, Nishimura $\mathrm{H}$ and Karasawa $\mathrm{K}$ : A prospective multicentre feasibility study of stereotactic body radiotherapy in Japanese patients with spinal metastases. Jpn J Clin Oncol 49(11): 9991003, 2019. PMID: 31665478. DOI: 10.1093/jjco/hyz130

19 Benedict SH, Yenice KM, Followill D, Galvin JM, Hinson W, Kavanagh B, Keall P, Lovelock M, Meeks S, Papiez L, Purdie T, Sadagopan R, Schell MC, Salter B, Schlesinger DJ, Shiu AS, Solberg T, Song DY, Stieber V, Timmerman R, Tomé WA, Verellen D, Wang L and Yin FF: Stereotactic body radiation therapy: the report of AAPM Task Group 101. Med Phys 37(8): 4078-4101, 2010. PMID: 20879569. DOI: 10.1118/1.3438081

20 Aljabab S, Vellayappan B, Vandervoort E, Bahm J, Zohr R, Sinclair J, Caudrelier JM, Szanto J and Malone S: Comparison of four techniques for spine stereotactic body radiotherapy: Dosimetric and efficiency analysis. J Appl Clin Med Phys 19(2): 160-167, 2018. PMID: 29417728. DOI: $10.1002 / \mathrm{acm} 2.12271$

Received October 15, 2021

Revised November 15, 2021 Accepted November 19, 2021 